\title{
Accuracy of Force Exertion in Each Demanded Value Based on Subjective Information and Laterality
}

\author{
Hiroki Aoki ${ }^{1, *}$, Shinichi Demura ${ }^{2}$, Shunsuke Yamaji ${ }^{3}$, Masashi Omoya ${ }^{4}$ \\ ${ }^{1}$ National Institute of Technology, Fukui College, General course, Fukui, Japan \\ ${ }^{2}$ Kanazawa University, Ishikawa, Japan \\ ${ }^{3}$ University of Fukui, Fukui, Japan \\ ${ }^{4}$ Chubu Yakuhin CO, LTD, Ishikawa, Japan \\ *Corresponding author: aoki@fukui-nct.ac.jp
}

\begin{abstract}
This study aimed to examine the direction of errors between demand values and exerted forces using submaximal grip strength based on laterality. Subjects were 100 healthy young males (age: $22.4 \pm 2.8$ years). After maximum grip strength (MGS) was measured, each subject exerted handgrip for each demanded value $(20 \%, 40 \%$, $60 \%$, and $80 \%$ ), twice with a 2-min rest between each trial. The means of all subjects were used for statistical analysis. Evaluation parameters were errors between exertion and demand values, which are relative values based on MGS. The Shapiro-Wilk test was performed to confirm the normality of the frequency distribution. It was confirmed that errors in demand values, except for $80 \%$ in the dominant hand, show a normal distribution. The result of twoway repeated measures analysis of variance (ANOVA; demanded values $\times$ dominant/non-dominant) and multiple comparisons showed that significant differences were found among demand values, except between $60 \%$ and $80 \%$ in the dominant hand, and the error was greater as follows: $20 \%>40 \%>60 \%$ and $80 \%$. The error in the nondominant hand was greater in the order of $20 \%, 40 \%, 60 \%$, and $80 \%$. A significant difference was found between errors of the dominant and non-dominant hands in demand values of $20 \%-60 \%$, and the error was smaller in the dominant hand. Test of independence for the frequency difference of exertion direction showed significant difference in all demand values except for $80 \% ; 20 \%$ and $40 \%$ did more in the positive direction (overexertion) in both hands, and $60 \%$ did more in the negative direction (underexertion) in the dominant hand and in the plus direction in the non-dominant hand. In conclusion, in the dominant hand, the error of grip force exertion toward demand values is greater in demand values under $60 \%$ and small grip vales are exerted. In contrast, in the nondominant hand, the error of grip force exertion toward demand values is greater in demand values under $80 \%$ and grip values are exerted largely in demand values under $60 \%$. Accuracy of force exertion differs among demand values, and laterality is found in low demand values of $20 \%-60 \%$.
\end{abstract}

Keywords: hand grip strength, accuracy, dominant hand, non dominant hand

Cite This Article: Hiroki Aoki, Shinichi Demura, Shunsuke Yamaji, and Masashi Omoya, "Accuracy of Force Exertion in Each Demanded Value Based on Subjective Information and Laterality." American Journal of Sports Science and Medicine, vol. 6, no. 1 (2018): 28-32. doi: 10.12691/ajssm-6-1-6.

\section{Introduction}

Handgrip strength has been evaluated mainly by maximum strength exertion, and great interest has been paid to methods for increasing the maximum strength and sustaining its exertion. However, Otsuki [1] reported that not only maximum strength, but also the ability to exert while adjusting strength are important. In addition, Halaney and Carey [2] reported that it is rare to exert maximum strength in daily life, and that submaximal strength is generally used. Nagasawa et al. [3] reported that the relationship between controlled force exertion and nervous function is high, and when exerting submaximal strength, motor skill is demanded. Submaximal strength exertion may be unstable and more difficult to maintain than maximum strength exertion [4]. Previous studies have used the absolute values of errors between demand and exertion values to evaluate controlled force exertion of grip strength. Exertion values greater (underexertion) and smaller (overexertion) than demand values have been seen as the same. In addition, Nagasawa et al. [3] have evaluated the accuracy of force exertion using the maximum or total of errors between demand and exertion values, but whether errors appear in the positive or negative direction has been ignored. Humans generally exert strength using the maximum strength as judgment criteria when exerting submaximal strength [5]. Therefore, a large error may occur in small demand values and be exerted superfluously. In contrast, error may be small in demand values that approach the maximum. On the other hand, a functional right and left difference called "laterality" exists in each body part with bilateral symmetry in humans $[4,6,7,8]$. This difference is particularly found in functions of fingers such as using a spoon or writing letters. This is considered to occur from a more preferential and frequent use of either hand in daily life 
activities. Until now, the lateral dominance of muscle function has frequently been reported. Noguchi et al. [9] reported that because a dominant hand is used more frequently in daily life, functions related to operation develop, and the difference between the hands becomes remarkable. In short, it is assumed that errors apart from demand values differ between dominant and non-dominant hands and may be large in the latter. Noguchi et al. [4] have examined laterality in controlled force exertion of grip strength. However, in controlled force exertion, subjects chase a moving target using eye-hand coordination. This exertion method differs from that of general force exertion. The laterality in submaximal strength exertions may be found in large demand values near maximal strength.

This study aimed to examine the direction of errors between demand and exertion values and the relation to laterality using submaximal grip strength (20\%, 40\%, 60\%, and $80 \%)$.

\subsection{Subjects}

Subjects were 100 healthy young males (age: $22.4 \pm 2.8$ years, height: $171.8 \pm 5.4 \mathrm{~cm}$, weight: $64.4 \pm 6.3 \mathrm{~kg}$, dominant handgrip strength: $51.1 \pm 8.3 \mathrm{~kg}$, non-dominant handgrip strength: $47.7 \pm 8.3 \mathrm{~kg}$ ). They all were judged to be right-hand dominant based on Demura's handedness inquiry [10]. Before measurements were taken, the purpose and procedure were explained in detail and informed consent was obtained from all participants. This experimental protocol was approved by the ethics committee on human experimentation of the Faculty of Human Science, Kanazawa University (Ref. No. 2012-02).

\subsection{Maximal Grip Strength Measurement}

Maximal grip strength (MGS) was measured using the Smedley Type Hand Dynamometer (YAGAMI INC., ED-D100R), which can measure strength of 0-979 N (99.9 kg) with a $\pm 2 \%$ accuracy as follows: The subjects exerted MGS with each hand, holding the arm straight down at the side of the trunk with an upright posture and looking straight ahead, as described by Crosby et al. [11] and Nagasawa and Demura [12]. They were instructed to exert force after a tester's signal. The MGS was measured twice in each hand with a 2-min rest time between the trials. MGS measured by each hand was used as the norm of the relative value of each demand value.

\subsection{Subjective Exerted Force Measurement}

Demand values selected for the subjective exertion force test were $20 \%, 40 \%, 60 \%$, and $80 \%$ of MGS. We did not give subjects feedback about their actual measured values. Two trials with a 2 -min rest were performed for each demanded value. The subjects were first divided into two groups to measure the dominant hand and the nondominant hand randomly. Measurement was performed in order of the demanded values of $20 \%, 40 \%, 60 \%$, and $80 \%$ according to the method of Noguchi et al. [13], which gradually enhances the demanded value. We covered the measurement device and strictly managed access to it so that subjects could not see exerted values.

\subsection{Evaluation Parameters}

The evaluation parameters were the errors between exertion and demand values on the basis of MGS.

\subsection{Data Analysis}

The Shapiro-Wilk test was used to confirm the normality of the frequency distribution of errors. Two-way repeated measures analysis of variance (ANOVA; demanded value $\times$ dominant/non-dominant hand) was used to reveal the differences among means of each condition. When a significant interaction or main effect was found, multiple comparison tests were performed using Tukey's honest significant difference method. Moreover, the frequency difference of the exertion direction according to demand values was examined using the test for independence, and the phi coefficient $(\varphi)$ was calculated. Statistical significance $(\alpha)$ was set at $\mathrm{p}<.05$.

\section{Results}

All variables showed very high intraclass correlation (ICC) of over 0.71 (ICC $=0.71-0.91$ ). Figure 1 shows the frequency distribution of $\mathrm{M}$ (mean) +0.5 SD(standard deviation) separations and the results of Shapiro-Wilk tests to confirm the normality. A normal distribution for all errors except $80 \%$ of the demand value for dominant hand was confirmed.

Table 1 shows the results of two-way ANOVA and multiple-comparison tests where we assumed that $80 \%$ of the demand value of the dominant hand is the normal distribution. Significant differences were found among the demand values except for between $60 \%$ and $80 \%$ in the dominant hand. The error was greater as follows: $20 \%>$ $40 \%>60 \%$ and $80 \%$. The mean value in $20 \%$ and $40 \%$ was above the demand value and in $60 \%$ and $80 \%$ was below.

On the other hand, the error in the non-dominant hand was greater (in the order of $20 \%, 40 \%, 60 \%$, and $80 \%$ of the demand values), and mean values were positive except for $80 \%$ of the demand value. A significant difference was found between errors of the dominant and non-dominant hands in demand values of $20 \%-60 \%$, and the error was smaller in the dominant hand. In addition, the same results were found when we conducted the Friedman test and Wilcoxon signed-rank test using the median values as representative values.

Figure 2 shows the results of the test of independence for the frequency difference of the exertion direction according to demand values. Significant difference was found in all demanded values except for $80 \%$; $20 \%$ and $40 \%$ showed more in the positive direction (overexertion) in both hands, and $60 \%$ showed a negative direction (underexertion) in the dominant hand and a positive direction (overexertion) in the non-dominant hand $(\varphi=$ $0.18-0.24)$. 

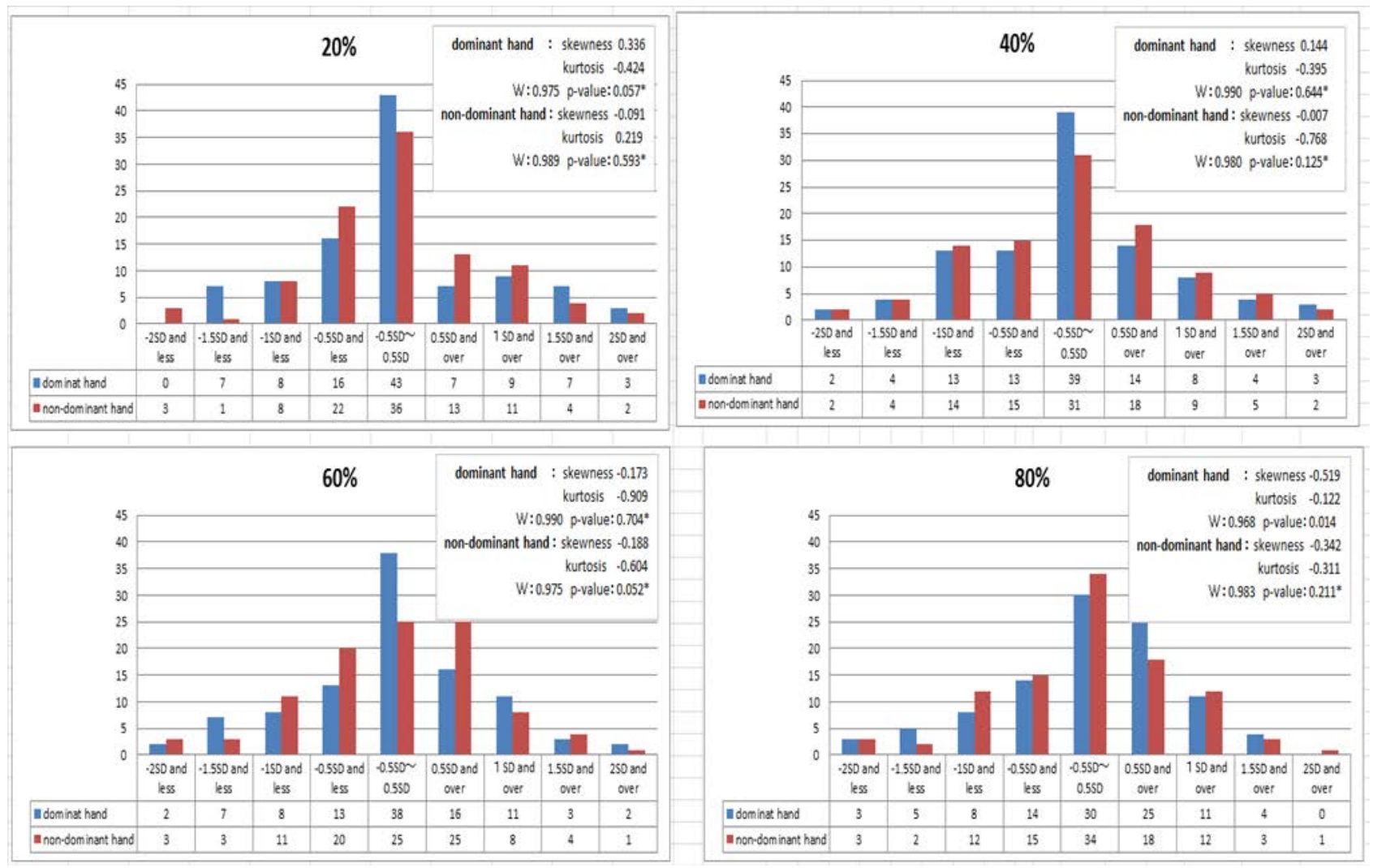

Figure 1. The results of frequency distribution of $\mathrm{M}$ (mean) $+0.5 \mathrm{SD}$ (standard deviation) separation and Shapiro-Wilk test

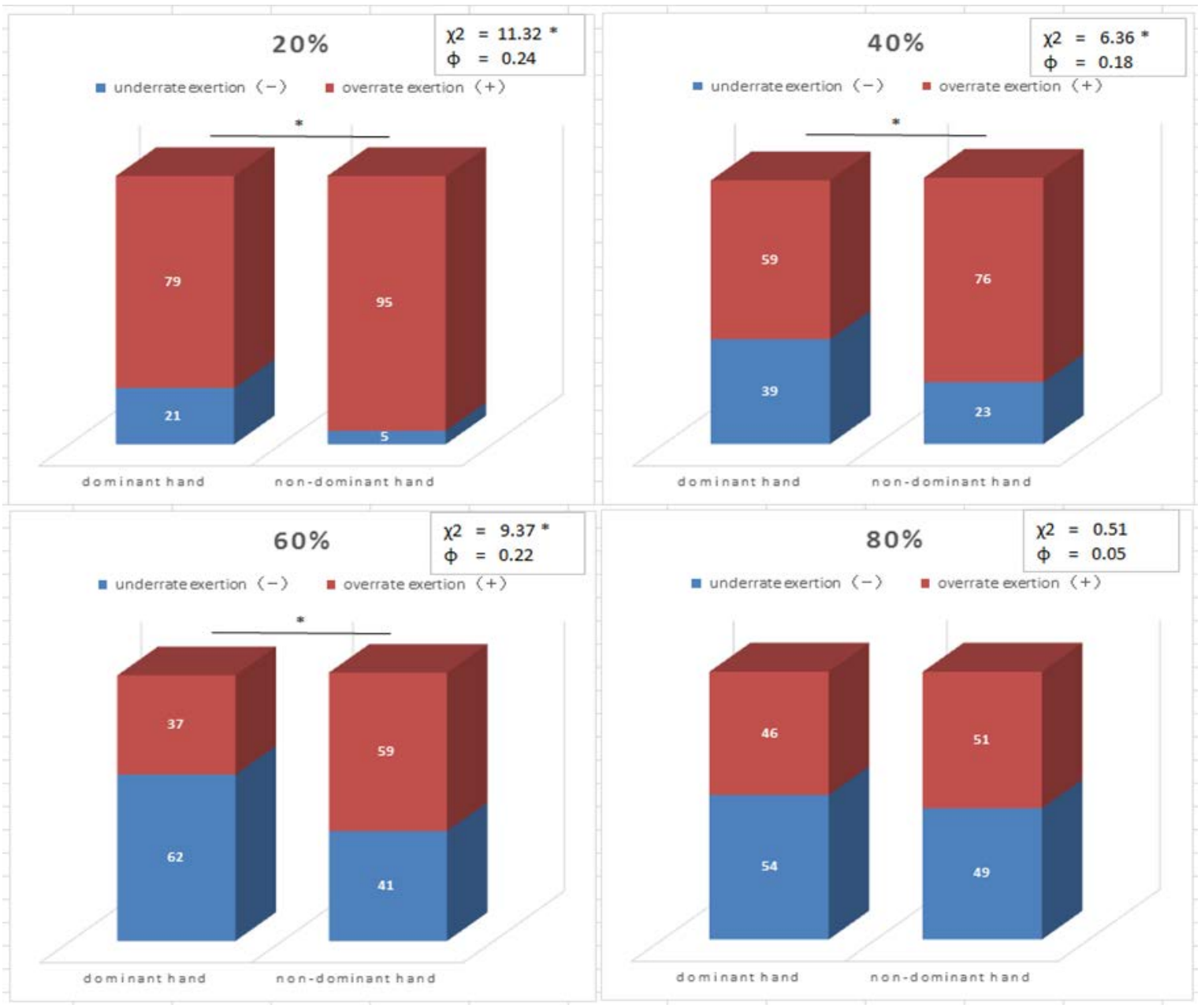

Figure 2. The results of test of independence for the frequency of the exertion direction according to demand value 
Table 1. The results of two-way ANOVA and multiple-comparison tests

\begin{tabular}{|c|c|c|c|c|c|c|c|c|c|}
\hline & $20 \%$ & $40 \%$ & $60 \%$ & $80 \%$ & & $\mathrm{~F}$ & $n^{2}$ & $\mathrm{P}$ & Post,hoc \\
\hline & Mean SD & Mean SD & Mean SD & Mean SD & $\mathrm{F} 1$ & $115.0 *$ & 0.54 & 0.00 & dominant hand:20>40>60,80\% \\
\hline dominant hand & $6.8 \quad 8.6$ & 1.98 .4 & $-2.6 \quad 9.1$ & $-1.8 \quad 8.3$ & $\mathrm{~F} 2$ & $82.2 *$ & 0.45 & 0.00 & morrdominant hand: $20>40>60>80 \%$ \\
\hline non-dominant hand & $13.0 \quad 9.2$ & 8.0 & $2.5 \quad 9.9$ & $-1.0 \quad 8.1$ & IN & $14.7 *$ & 0.13 & 0.00 & $20,40,60 \mathrm{~K}$ : non-domin ant hand $>$ dominant hand \\
\hline
\end{tabular}

\section{Discussion}

Frequency distribution of the error was confirmed to be normal in all demand values except for $80 \%$ in the dominant hand. In the case of voluntary force exertion, Ohtsuki et al. [5] posited that we feel the sense of exerting the maximal voluntary contraction as internal information, and thus better control our subjective exertion force. Aoki \& Demura (in press) [14] reported that the accuracy in the subjective force exertions of elbow flexion is higher in demand values near maximal force in both dominant and non-dominant arms. Stevens and Mack [15] reported that errors in the low demand values decrease according to power law as the output intensity becomes high. The results of this study also confirmed that errors decrease according to power law as demand values increase. In short, the accuracy of strength exertion may stabilize at approximately $60 \%$ in the dominant hand. In addition, the strength exertion may exert less as the demand value approaches maximum strength, because a mean error showed a negative direction after the $60 \%$ demand value.

However, the non-dominant hand had a tendency of error that looked similar to the dominant hand, but errors in $20 \%-60 \%$ demand values were greater than those in the dominant hand. As mentioned above, the power law to which an error decreases as the demand value increases appears to have more of an effect on the non-dominant hand, and accuracy may be inferior to that in the dominant hand. In addition, an average error appeared in the negative direction in the non-dominant hand only for $80 \%$ of the demand value. This confirmed that strength in the non-dominant hand is exerted to a strength of approximately $80 \%$ of the demand value. Sadamoto and Ohthuki [16] reported that the accuracy of the program formed by the past movement learning and experience and the output mechanism converting the program to output force are contained in output coordinates. Noguchi et al. [9] reported that because of being used more frequently than usual, the functions related to operation develop more strongly in the dominant hand, and the difference between the hands becomes remarkable. In short, the present results, in which laterality was found in low demand values such as $20 \%-60 \%$ and the non-dominant hand proved inferior in accuracy of force exertion, may be largely affected by unbalanced use of the dominant hand in everyday life, as the abovementioned studies showed.

We also noted the direction of exerted errors and examined the frequency of errors exerted in the positive (overexertion) and negative (underexertion) directions for each demand value. In the results, $20 \%$ and $40 \%$ of the demand values showed a high frequency in the positive direction (overexertion) in both hands. At $60 \%$ of the demand value, there was more error in the negative direction (underexertion) in the dominant hand and more in the positive direction (overexertion) in the non-dominant hand. From the above, it can be inferred that the difference between dominant and non-dominant hands becomes remarkable at approximately $60 \%$ (about half of maximum strength), and when considering that more errors were exerted in the positive direction for $20 \%$ and $40 \%$ of the demand values in the non-dominant hand, the dexterous nature of the non-dominant hand may decrease from approximately $60 \%$ demand value. Sainburg and Kalakanis [17] reported that a dominant hand differs from a non-dominant hand in the mechanism of adjusting strength exertion and motion of joints. The difference in physiological strategy like the above becomes remarkable at approximately $60 \%$, manifesting as the difference between dominant and non-dominant hands. However, there was no difference between dominant and non-dominant hands at $80 \%$ of the demand value, and the errors in both hands appeared in the negative direction (underexertion). According to Ohthuki [5], humans judge an exertion value on the basis of MGS. By having a standard that becomes an index of exertion value, the accuracy of output increases regardless of dominant or non-dominant hands. In the future, when performing adjustment of force exertion to some demand values, we intend to examine whether the accuracy and reproducibility of exertion force is enhanced by performing prior practice of the intensity corresponding to their demand values.

\section{Conclusion}

In the dominant hand, the error of grip force exertion toward demand values is greater in demand values under $60 \%$ and small grip vales are exerted. In contrast, in the non-dominant hand, the error of grip force exertion toward demand values is greater in demand values under $80 \%$ and grip values are exerted largely in demand values under $60 \%$. Accuracy of force exertion differs among demand values, and laterality is found in lower demand values of $20 \%-60 \%$.

\section{References}

[1] Ohtsuki T. (1988). "The science of the skillful,” Asakurasyoten, Tokyo, 123-144.

[2] Halaney, M.E. and Carey, J.R. "Tracking ability of hemiparetic and healthy subjects," Physical Therapy, 69, 342-348, 1989.

[3] Nagasawa, Y., Demura, S. and Kitabayashi, T., "Concurrent validity of tests to measure the coordinated exertion of force by computerized target pursuit," Percept Mot Skills, 98, 551-560. 2004.

[4] Noguchi, T., Demura, S. and Aoki, H., "Superiority of the dominant and nondominant hands in static strength and controlled force exertion,” Perceptual and Motor Skills, 109, 339-346. 2009. 
[5] Ohtsuki, T., "Skillful of Brain and Voluntary Exercise," Journal of Health, Physical education and Recreation, 46, 444-446. 1996.

[6] Dolcos, F., Rice, HJ. and Gabeza, R., "Hemispheric asymmetry and aging right hemisphere decline or asymmetry reduction," Neurosci Biobehav, 26, 819-825.2002.

[7] Bohannon, R., "Grip strength: a summary of studies comparing dominant and non dominant limb measurements," Percept Mot Skills, 96, 728-730. 2003.

[8] Roy, E., Bryden, P. and Gavill, S., "Hand differences in pegboard performance through development,” Brain Cogn, 53, 315-317. 2003.

[9] Noguchi, T., Demura,S., Nagasawa, Y. and Uchiyama, M., "The practice effect and its difference of the pursuit rotor test with the dominant and non-dominant hands," The Journal of Physiological Anthropology and Applied Human Science, 24, 589-93. 2005.

[10] Demura, S., Sato, S. and Nagasawa, Y., "Re-examination of useful items for determining hand dominance,” Medica Italiana Archivio per le Scienze Mediche, 169-177. 2009.

[11] Crosby, C.A., Wehbe, M.A. and Mawr, B., "Hand strength: normative values” The Journal of hand surgery, 19, 665-670. 1994.
[12] Nagasawa, Y. and Demura, S., "Development of an apparatus to estimate coordinated exertion of force" Perceptual and Motor Skills, 94, 899-913. 2002.

[13] Noguchi, T., Demura, S. and Omoya, M., "Accuracy of Force Exertion in Response to Demanded Forces Based on Subjective Information and Laterality,” American Journal of Sports Science and Medicine, 2, 190-193. 2014

[14] Aoki, H., Demura, S., "Laterality and accuracy of force exertion in elbow flexion," ape, in press.

[15] Stevens, JC. And Mack, JD., "Scales of apparent force,” Journal of Experimental Psychology, 68, 405-413.1959.

[16] Sadamoto, T. and Ohtsuki, T., "Accuracy of Output Control in Jumping: Characteristics in Grading and Reproduction of Distance,” Japan Journal of Physical Education, 22, 215-229. 1977.

[17] Sainburg, RL. and Kalakanis D., "Differences in control of limb dynamics during dominant and nondominant arm reaching," Journal of Neurophysiology, 83, 2661-2675. 2000. 